\title{
Investigation of nonlinear restoring stiffness in dynamic analysis of tension leg platforms
}

\author{
I. Senjanović*, M. Tomić, S. Rudan \\ ${ }^{a}$ University of Zagreb, Faculty of Mechanical Engineering and Naval Architecture, I. Lučića 5, 10000 Zagreb, Croatia
}

\section{A R T I C L E I N F O}

\section{Article history:}

Received 20 December 2012

Revised 22 April 2013

Accepted 23 April 2013

\section{Keywords:}

Tension leg platform

Nonlinear stiffness

Force equilibrium

Energy balance

FEM

\begin{abstract}
A B S T R A C T
Shortcomings of the traditional stiffness matrix in dynamic analysis of TLPs, derived by considering equilibrium of forces, are pointed out, as well as dilemma concerning consistency of the recently presented matrix based on energy balance. New stiffness matrix is derived by utilizing both force equilibrium approach, with algebraic averaging and root mean square of tendon forces, and energy balance approach for large surge and sway. Yaw is treated as a small and large displacement. Static numerical analysis is performed for all six cases by imposing surge force and yaw moment. The obtained results are compared with those of FEM analysis, and useful conclusion is drawn, which can be used for improvement of uncoupled mathematical model of TLPs.
\end{abstract}

(c) 2013 Elsevier Ltd. All rights reserved.

\section{Introduction}

Tension leg platform (TLP) is classified as a compliant offshore structure, i.e. a semi-submersible one attached to the sea bottom by vertical pretensioned tendons or tethers, [1]. It is ordinary used for deep water oil operations. At the beginning the installed depth was $147 \mathrm{~m}$, Hutton (1984), and nowadays it reaches much higher values, for instance $1425 \mathrm{~m}$, Magnolia (2005). The consisting parts of a TLP are: pontoon, columns and deck with drilling equipment, [2].

TLP motion in waves is nonlinear due to nonlinear restoring stiffness and damping, [3]. The tendons make TLPs more mobile in horizontal than in vertical plane and ensure almost horizontal position of the working area. Vertical excitation is caused by the first order wave forces, while dominant horizontal excitation is due to the second order wave forces, [4]. Vertical response, i.e. heave, roll and pitch, has high natural frequencies due to high axial tendon stiffness. Natural frequencies of horizontal motion, i.e. surge, sway and yaw, are much lower due to tendon geometric stiffness and can easily fall into resonance with the forcing frequency.

Restoring stiffness plays very important role in TLPs dynamic behavior. Horizontal motion is nonlinear since stiffness is function of surge, sway and yaw. Stiffness of vertical motion is almost linear and depends on platform offset, which causes setdown, as position

\footnotetext{
* Corresponding author. Tel.: +385 16168 142; fax: +385 16156940.

E-mail address: ivo.senjanovic@fsb.hr (I. Senjanović).
}

parameter. Setdown is one of very important design parameters for limiting platform immersion [5].

Nowadays, the secant stiffness matrix introduced in [6] and slightly modified in $[7,8]$ is still widely used for dynamic analysis of TLPs. Its formulation is based on equilibrium of restoring forces due to large displacements. One finite displacement is imposed while the others are restrained. Asymmetric stiffness matrix for six d.o.f. is established with respect to the center of gravity. Shortcomings of that formulation is that stiffness elements of surge, sway and yaw depend on tendon axial stiffness instead of a buoyancy increase due to setdown, as a hydrostatic spring. The former stiffness is much larger than the latter, and since setdown is not taken into account, implication is excessively large stiffness of horizontal motions. By considering equilibrium due to particular displacements, coupling motions is not taken into account. These problems are analysed in details in [9].

Recently, another formulation of nonlinear restoring stiffness for TLPs is presented in [10], specifying also the shortcomings of the above mentioned traditional one. The stiffness matrix is derived by the energy approach and employing Lagrange's equations. Since the tendon setdowns are different due to coupling of surge and sway with yaw, the platform is considered as independent quadrants, which follow the tendon top motion. Potential energy of the system is established under that assumption, and its first derivatives per displacements give the restoring forces, i.e. secant stiffness matrix. The shortcomings of the traditional stiffness are overcome, i.e. stiffness of horizontal motion depends on platform 
hydrostatics, and coupling between surge, sway and yaw is captured.

Dynamic analysis of TLP is performed by the uncoupled and fully coupled models [11]. In the former case platform is considered as a rigid body without tendon influence, and linear restoring is applied. If nonlinear damping is linearized problem can be solved in frequency domain. That advantage of reduction of computing time is paid by decreased accuracy.

Fully coupled model is actually 3D FEM model of platform and tendons adapted to large displacements. Due to mechanical and hydrodynamical nonlinearity problem is analysed in time domain. Nonlinear equation of motion can be linearized that makes some difficulties and limitations.

Motivated by the fact that the uncoupled dynamic analysis of TLPs is widely performed by using linear or an inadequate nonlinear restoring stiffness matrix, research for consistent stiffness is undertaken [9]. The force equilibrium approach is employed and stiffness similar to that in [10], determined by the energy approach, is obtained. New stiffness elements are the same for surge, sway and yaw, but without some additional coupling terms present in the energy formulation [10]. Hence, there is doubt which of the two stiffness formulations is a proper one.

In order to overcome that dilemma nonlinear restoring stiffness is derived in this paper by employing both the force equilibrium and energy balance approach for large surge and sway, as well as yaw motion that was not the case in the previous considerations, [9] and [10]. Then, the obtained stiffness expressions are reduced for the case of small yaw.

In order to evaluate two different restoring stiffness formulations, static response of a TLP platform exposed to surge force and yaw moment is analysed, treating yaw both as a small and large quantity. The obtained results are compared to those determined by FEM analysis that leads to some interesting conclusion.

\section{Stiffness based on equilibrium of forces}

\subsection{Large translation and rotation}

A double symmetric rectangular TLP, with four tendons and main dimensions shown in Fig. 1, is considered. The platform is exposed to large surge, sway and yaw, $\delta_{x}, \delta_{y}$ and $\varphi$, which are common for all tendons, Fig. 2. Trajectory of the tendon top due to yaw is circular arc, $r \varphi$, where $r$ is the tendon radial distance from the platform centroid. The tendon final offset is determined with the secant displacement, Fig. 2

$\delta_{\varphi}=2 r \sin \frac{\varphi}{2}$.

According to Fig. 2 the tendon top coordinates in the local coordinate system read

$L_{x}^{n}=\delta_{x}-\Delta_{x}^{n}=\delta_{x}-\delta_{\varphi} \sin \left(\theta_{n}+\frac{\varphi}{2}\right)$,

$L_{y}^{n}=\delta_{y}+\Delta_{y}^{n}=\delta_{y}+\delta_{\varphi} \cos \left(\theta_{n}+\frac{\varphi}{2}\right)$,

$L_{z}^{n}=\sqrt{L^{2}-\left(L_{x}^{n}\right)^{2}-\left(L_{y}^{n}\right)^{2}}$

$$
=L\left\{1-\frac{1}{L^{2}}\left(\delta_{x}^{2}+\delta_{y}^{2}+\delta_{\varphi}^{2}\right)+\frac{2 \delta_{\varphi}}{L^{2}}\left[\delta_{x} \sin \left(\theta_{n}+\frac{\varphi}{2}\right)-\delta_{y} \cos \left(\theta_{n}+\frac{\varphi}{2}\right)\right]\right\}^{\frac{1}{2}},
$$

where $L$ is tendon length and $\theta_{n}, n=1,2,3,4$, is tendon central angle.

Components of the tendon tension forces $T_{n}$ in an offset position are proportional to the tendon top coordinates
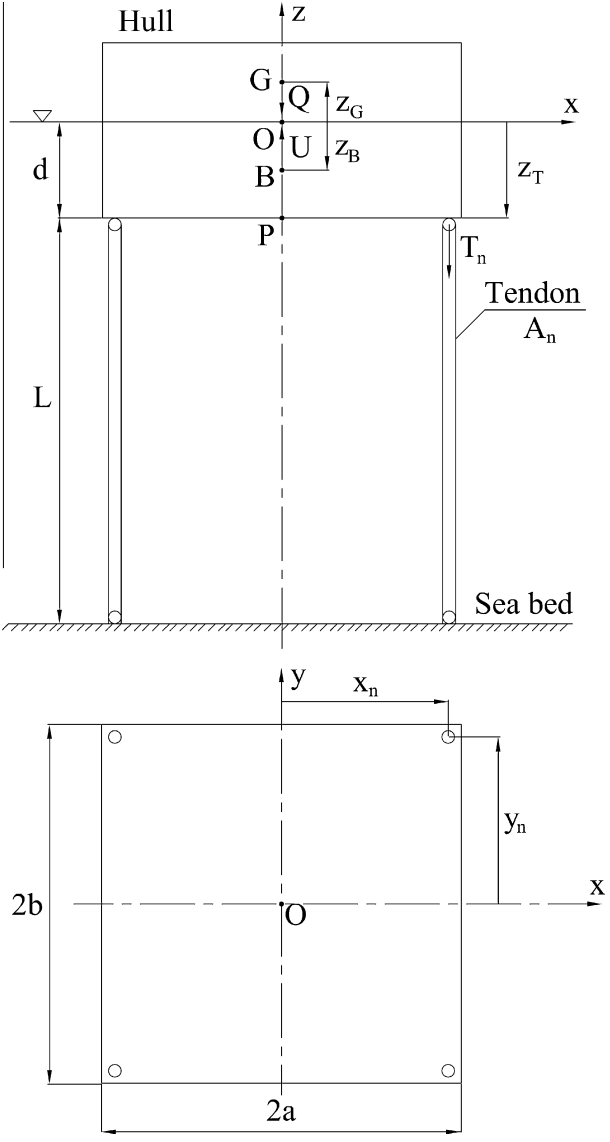

Fig. 1. Double symmetric TLP.

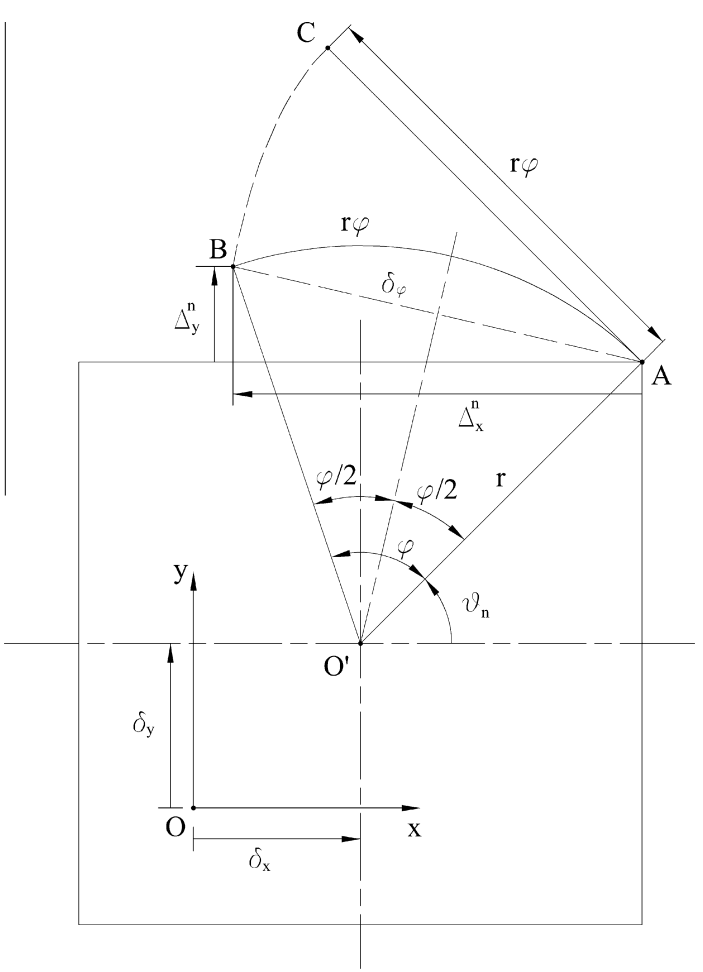

Fig. 2. Large surge, sway and yaw. 
$T_{x}^{n}=\frac{T_{n}}{L}\left[\delta_{x}-\delta_{\varphi} \sin \left(\theta_{n}+\frac{\varphi}{2}\right)\right]$,

$T_{y}^{n}=\frac{T_{n}}{L}\left[\delta_{y}+\delta_{\varphi} \cos \left(\theta_{n}+\frac{\varphi}{2}\right)\right]$,

$T_{z}^{n}=\frac{T_{n}}{L} L_{z}^{n}$

Total tendon force acting on platform is $T=\sum_{n=1}^{N} T_{n}$. Its components according to (5)-(7) read

$T_{x}=\sum_{n=1}^{N} T_{x}^{n}=\frac{T}{L} \delta_{x}$

$T_{y}=\sum_{n=1}^{N} T_{y}^{n}=\frac{T}{L} \delta_{y}$

$T_{z}=\sum_{n=1}^{N} T_{z}^{n}=\frac{T}{L} \frac{1}{N} \sum_{n=1}^{N} L_{z}^{n}$.

Horizontal components $T_{x}$ and $T_{y}$ depend only on horizontal displacements $\delta_{x}$ and $\delta_{y}$, respectively, since the trigonometric functions vanish from (5) and (6) upon summation owing to the platform double symmetry. However, values of $L_{z}^{n}$ are different in case of yaw. In order to make summation of $L_{z}^{n}$ in (10) possible let us expand $L_{z}^{n}$ into power series. Since $\sqrt{1-\varepsilon}=1-\frac{1}{2} \varepsilon-\frac{1 \cdot 1}{2 \cdot 4} \varepsilon^{2}-\cdots$ one can write

$L_{z}=\frac{1}{N} \sum_{n=1}^{N} L_{z}^{n}=L\left(1-f_{1}-f_{2}\right)$,

where

$f_{1}=\frac{1}{2 L^{2}}\left(\delta_{x}^{2}+\delta_{y}^{2}+\delta_{\varphi}^{2}\right), \quad f_{2}=\frac{1}{2} f_{1}^{2}+f_{3}$,

$f_{3}=\frac{2}{L^{4}}\left[\delta_{x}^{2}\left(b^{2} \cos ^{2} \frac{\varphi}{2}+a^{2} \sin ^{2} \frac{\varphi}{2}\right)+\delta_{y}^{2}\left(a^{2} \cos ^{2} \frac{\varphi}{2}+b^{2} \sin ^{2} \frac{\varphi}{2}\right)\right.$

$\left.+\delta_{x} \delta_{y}\left(b^{2}-a^{2}\right) \sin \varphi\right] \sin ^{2} \frac{\varphi}{2}$.

Functions $f_{1}$ and $f_{2}$ are the first and second order terms, respectively. Relatively simple expressions are obtained since trigonometric functions of argument $\theta_{n}$ with odd exponent vanish upon summation. Also, relation (1) for $\delta_{\varphi}, \cos ^{2} \theta_{n}=(a / r)^{2}$ and $\sin ^{2} \theta_{n}=(b / r)^{2}$ are employed. In that way angles $\theta_{n}$ disappear from $L_{z}$. Differences $L_{z}^{n}-L_{z}$ cause some small platform roll and pitch.

The tendon offsets cause platform setdown $\delta^{S}=L-L_{z}$, and consequently additional buoyancy $\Delta U$ and tendon forces $\Delta T_{n}$. The resulting additional tendon force can be determined from the equilibrium of the vertical forces

$U+\Delta U=Q+\frac{1}{L} \sum_{n=1}^{N}\left(T_{n}+\Delta T_{n}\right) L_{z}^{n}$,

where $Q$ is the platform weight. The second term in (14) is written according to (10). The increased buoyancy reads

$\Delta U=\rho g A_{W L} \delta^{s}$,

where $A_{W L}$ is the waterplane area. By taking floating condition $T=U-Q$ into account and substituting (15) into (14), one finds for the total additional tendon force

$\Delta T=\frac{1}{L_{z}} \sum_{n=1}^{N} \Delta T_{n} L_{z}^{n}=\frac{1}{L_{z}}\left(T+\rho g A_{W L} L\right) \delta^{s}$.

Horizontal component of the total tendon force (8) is increased, and has to be equal to the external force, $T_{x}=F_{x}$, i.e.

$F_{x}=\frac{1}{L}(T+\Delta T) \delta_{x}=\tilde{K}_{0} \delta_{x}$, where $\widetilde{K}_{0}$ is nonlinear secant stiffness. By substituting (16) into (17) yields

$\widetilde{K}_{0}=\frac{1}{L_{z}}\left(T+\rho g A_{W L} \delta^{s}\right)=\frac{C}{L_{z}}-\rho g A_{W L}$,

where

$C=T+\rho g A_{W L} L$.

Analogously, the sway force reads $T_{y}=F_{y}=\widetilde{K}_{0} \delta_{y}$.

The yaw moment is caused by the horizontal tendon forces

$M_{z}=-\sum_{n=1}^{N}\left(T_{x}^{n}+\Delta T_{x}^{n}\right) y_{n}+\sum_{n=1}^{N}\left(T_{y}^{n}+\Delta T_{y}^{n}\right) x_{n}$

where $x_{n}=r \cos \theta_{n}$ and $y_{n}=r \sin \theta_{n}$ are the tendon top coordinates, Fig. 1. By employing (5) and (6) one finds

$M_{z}=\frac{r}{L} \sum_{n=1}^{N}\left(T_{n}+\Delta T_{n}\right)\left(-\delta_{x} \sin \theta_{n}+\delta_{y} \cos \theta_{n}+\delta_{\varphi} \cos \frac{\varphi}{2}\right)$.

Trigonometric functions of $\theta_{n}$ vanish upon summation and taking (1) for $\delta_{\varphi}$ into account one arrives at

$M_{z}=\frac{r^{2}}{L}(T+\Delta T) \sin \varphi=\widetilde{K}_{0} r^{2} \varphi$.

Since according to $(17)(T+\Delta T) / L=\widetilde{K}_{0}$, the yaw stiffness reads

$\widetilde{K}_{66}=r^{2} \widetilde{K}_{0} \frac{\sin \varphi}{\varphi}$.

Function $\sin \varphi / \varphi$ represents reduction of arm of the tendon horizontal forces. For hypothetically large value $\varphi=\pi$, the arm is zero.

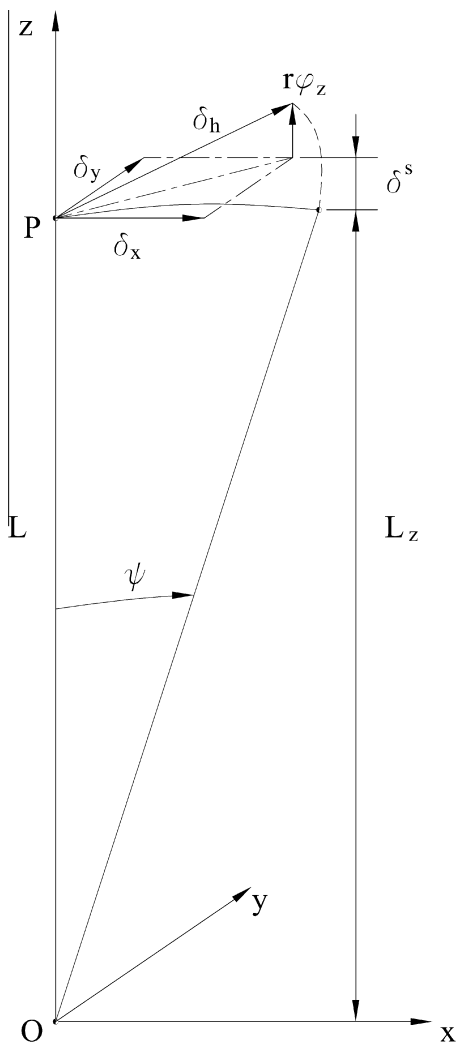

Fig. 3. Construction of setdown in offset plane 


\subsection{Large translation and small rotation}

If only the first two terms of expansion (11) for $L_{z}$ are taken into account, the setdown reads

$\delta^{S}=L-L_{z}=L f_{1}=\frac{1}{2 L}\left(\delta_{x}^{2}+\delta_{y}^{2}+\delta_{\varphi}^{2}\right)$.

It can be constructed as shown in Fig. 3, where $\delta_{h}$ and $\psi$ is virtual horizontal displacement and offset angle, respectively.

Furthermore, if yaw angle $\varphi$ is small then according to (1) $\delta_{\varphi}=r \varphi$ and

$\delta^{s}=\frac{1}{2 L}\left(\delta_{x}^{2}+\delta_{y}^{2}+r^{2} \varphi^{2}\right)$

In that case the yaw stiffness (23) is also simplified, $\widetilde{K}_{\varphi}=r^{2} \widetilde{K}_{0}$. It is obvious that stiffness in any horizontal direction is the same.

\section{Stiffness based on balance of potential energy}

\subsection{Large translation and rotation}

The following forces act on platform: $U, Q, T, \Delta T$ and $\Delta U$. Tendon elongation $\Delta L_{n}$ due to tendon forces $T_{n}+\Delta T_{n}$ is very small comparing to the platform setdown and therefore is ignored. Work of the external vertical forces $U-Q=T$ is done in the way of setdown, $\delta^{S}$. Buoyancy variation $\Delta U$ is internal force increasing proportionally to the setdown. Hence, one can specify the platform potential energy as

$V=T \delta^{s}+\frac{1}{2} \rho g A_{W L}\left(\delta^{s}\right)^{2}$

The platform vertical coordinate $L_{z}$ is defined by (11), and accordingly the setdown $\delta^{s}=L-L_{z}$ reads

$\delta^{s}=L\left(f_{1}+f_{2}\right)$,

where $f_{1}$ and $f_{2}$ are specified with (12) and (13), respectively. $L_{z}$ can also be determined as the quadratic mean of the tendon vertical coordinates (4), i.e.

$L_{z}^{2}=\frac{1}{N} \sum_{n=1}^{N}\left(L_{z}^{n}\right)^{2}$

Since the trigonometric functions of angles $\theta_{n}$ in $\left(L_{z}^{n}\right)^{2}$ vanish upon summation, yields

$L_{z}^{2}=L^{2}\left(1-2 f_{1}\right)$

On the other side, relation $\delta^{s}=L-L_{z}$ gives

$\left(\delta^{S}\right)^{2}=L^{2}-2 L L_{z}+L_{z}^{2}$.

By substituting (11) and (29) into (30) one arrives at

$\left(\delta^{s}\right)^{2}=2 L^{2} f_{2}$.

Finally, by employing (27) and (31), Eq. (26) can be presented in the form

$V=T L f_{1}+C L f_{2}$,

where function $f_{2}$ of the second order is transferred from the first term to the second one.

Since function $f_{2}$ consists of two terms, Eq. (12), yields

$V=T L f_{1}+\frac{1}{2} C L f_{1}^{2}+C L f_{3}$,

Restoring forces are obtained as derivatives of the potential energy per particular displacement. Hence, for the secant stiffness of surge, sway and yaw one can write

$$
\begin{aligned}
& \widetilde{K}_{11}=\frac{L}{\delta_{x}}\left(T \frac{\partial f_{1}}{\partial \delta_{x}}+C \frac{\partial f_{2}}{\partial \delta_{x}}\right), \\
& \widetilde{K}_{22}=\frac{L}{\delta_{y}}\left(T \frac{\partial f_{1}}{\partial \delta_{y}}+C \frac{\partial f_{2}}{\partial \delta_{y}}\right), \\
& \widetilde{K}_{66}=\frac{L}{\varphi}\left(T \frac{\partial f_{1}}{\partial \varphi}+C \frac{\partial f_{2}}{\partial \varphi}\right) .
\end{aligned}
$$

Derivatives of functions $f_{1}$ and $f_{2}$. Eqs. (12) and (13), are the following:

$$
\begin{aligned}
\frac{\partial f_{1}}{\partial \delta_{x}}= & \frac{\delta_{x}}{L^{2}}, \frac{\partial f_{1}}{\partial \delta_{y}}=\frac{\delta_{y}}{L^{2}}, \frac{\partial f_{1}}{\partial \varphi}=\frac{r^{2}}{L^{2}} \sin \varphi \\
\frac{\partial f_{2}}{\partial \delta_{x}}= & \frac{1}{L^{2}} f_{1} \delta_{x}+\frac{4}{L^{4}} f_{3} \delta_{x} \sin ^{2} \frac{\varphi}{2}, \\
\frac{\partial f_{2}}{\partial \delta_{y}}= & \frac{1}{L^{2}} f_{1} \delta_{y}+\frac{4}{L^{4}} f_{4} \delta_{y} \sin ^{2} \frac{\varphi}{2}, \\
\frac{\partial f_{2}}{\partial \varphi}= & \frac{r^{2}}{L^{2}} f_{1} \sin \varphi+\frac{1}{L^{4}}\left[f_{4} \delta_{x}^{2}+f_{5} \delta_{y}^{2}+\left(b^{2}-a^{2}\right) \delta_{x} \delta_{y} \sin \varphi\right] \sin \varphi \\
& +\frac{b^{2}-a^{2}}{L^{4}}\left[\left(\delta_{y}^{2}-\delta_{x}^{2}\right) \sin \varphi+2 \delta_{x} \delta_{y} \cos \varphi\right] \sin ^{2} \frac{\varphi}{2},
\end{aligned}
$$

where $f_{1}$ is represented with (18) and

$f_{4}=b^{2} \cos ^{2} \frac{\varphi}{2}+a^{2} \sin ^{2} \frac{\varphi}{2}$,

$f_{5}=a^{2} \cos ^{2} \frac{\varphi}{2}+b^{2} \sin ^{2} \frac{\varphi}{2}$.

\subsection{Large translation and small rotation}

If yaw angle is small $\sin \varphi \approx \varphi, \cos ^{2} \frac{\varphi}{2} \approx 1$ and $\sin ^{2} \frac{\varphi}{2} \approx 0$, the stiffness elements, Eqs. (34)-(36), take quite simple form

$\widetilde{K}_{11}=\widetilde{K}_{0}+\frac{C}{L^{3}} b^{2} \varphi^{2}$,

$\widetilde{K}_{22}=\widetilde{K}_{0}+\frac{C}{L^{3}} a^{2} \varphi^{2}$,

$\widetilde{K}_{66}=r^{2} \widetilde{K}_{0}+\frac{C}{L^{3}}\left[b^{2} \delta_{x}^{2}+a^{2} \delta_{y}^{2}+\left(b^{2}-a^{2}\right) \delta_{x} \delta_{y}\right]$,

where $\widetilde{K}_{0}$ is specified with Eq. (34). Last term in (53) in case of a square platform is zero, while for a rectangular one its value depends on the platform aspect ratio. If that term is ignored due to small value, elements $\widetilde{K}_{i i}, i=1,2,6$, are identical to those presented in [10]. They are derived directly for small yaw angle and utilizing the energy approach based on the assumption that each platform quarter moves separately together with the corresponding tendon.

\section{Stiffness based on root means square of tendon forces}

\subsection{Large translation and rotation}

In spite of the fact that stiffness elements shown in the previous sections are consistently derived based on equilibrium of forces and energy balance, different formulas are obtained. The reason for that is use of the algebraic and quadratic mean of the vertical tendon forces, expressed with coordinates $L_{z}^{n}$, Eq. (10), in the former and latter case, respectively. Consequence of natural application of the algebraic mean on potential energy is investigated in Appendix.

In addition it is interesting to apply the quadratic mean for vertical tendon forces in the force equilibrium approach. One can start with rigorous expression for $\widetilde{K}_{0}$, Eq. (18). Since $L_{z}=L-\delta^{s}$ and by employing relation $(1-\varepsilon)^{-1} \approx 1+\varepsilon$, yields 
Table 1

Main particulars of the ISSC TLP.

\begin{tabular}{lll}
\hline Parameters & Symbol & Value \\
\hline Column (tendon) spacing & $2 a=2 b$ & $86.25 \mathrm{~m}$ \\
Column diameter & $D_{C}$ & $16.87 \mathrm{~m}$ \\
Pontoon width & $w$ & $7.50 \mathrm{~m}$ \\
Pontoon height & $d$ & $10.50 \mathrm{~m}$ \\
Draft & $-z_{T}$ & $35.00 \mathrm{~m}$ \\
Waterplane area & $A_{w l}$ & $894 \mathrm{~m}^{2}$ \\
Displacement & $U$ & $5.346 \times 10^{5} \mathrm{kN}$ \\
Weight & $Q$ & $3.973 \times 10^{5} \mathrm{kN}$ \\
Total tendon pretension & $T$ & $1.373 \times 10^{5} \mathrm{kN}$ \\
Longitudinal metacentric height & $\overline{M_{L} G}$ & $6.0 \mathrm{~m}$ \\
Transverse metacentric height & $\overline{M_{T} G}$ & $6.0 \mathrm{~m}$ \\
Platform mass & $m$ & $40.5 \times 10^{3} \mathrm{t}$ \\
Roll mass moment of inertia & $J_{x}^{G}$ & $82.37 \times 10^{6} \mathrm{t} \mathrm{m}$ \\
Pitch mass moment of inertia & $J_{y}^{G}$ & $82.37 \times 10^{6} \mathrm{t} \mathrm{m}{ }^{2}$ \\
Yaw mass moment of inertia & $J_{z}^{G}$ & $98.07 \times 10^{6} \mathrm{t} \mathrm{m}{ }^{2}$ \\
Vertical position of COG above keel & $\overline{P G}$ & $38.0 \mathrm{~m}$ \\
Vertical position of COB above keel & $\overline{P B}$ & $22.3 \mathrm{~m}$ \\
Length of mooring tendons & $L$ & $415.0 \mathrm{~m}$ \\
Tendon cross-section area & $A$ & $1.6 \mathrm{~m}{ }^{2}$ \\
Vertical stiffness of combined tendons & $\frac{E A}{L}$ & $0.813 \times 10^{6} \mathrm{kN} / \mathrm{m}$ \\
Roll and pitch effective stiffness & $\frac{E I_{x}}{L}, \frac{E I_{y}}{L}$ & $1.501 \times 10^{9} \mathrm{kNm} / \mathrm{rad}$ \\
Young's modulus & $E$ & $2.1 \times 10^{8} \mathrm{kN} / \mathrm{m}^{2}$ \\
& & \\
& $E$ &
\end{tabular}

$\widetilde{K}_{0}=\frac{T}{L}+\frac{C}{L^{2}} \delta^{s}$.

For setdown $\delta^{s}$ quadratic mean (31), i.e. root mean square, can be used that arrives at

$\widetilde{K}_{R M S}=\frac{T}{L}+\frac{C}{L^{2}} \delta_{R M S}^{S}$,

where

$\delta_{R M S}^{s}=L \sqrt{2 f_{2}}$.

By employing the second of Eqs. (12) the stiffness is presented in the final form

$\widetilde{K}_{R M S}=\frac{T}{L}+\frac{C}{L} \sqrt{f_{1}^{2}+2 f_{3}}$.

\subsection{Large translation and small rotation}

If yaw angle is small one finds from Eqs. (12) and (13)

$f_{1}=\frac{1}{2 L^{2}}\left(\delta_{x}^{2}+\delta_{y}^{2}+r^{2} \varphi^{2}\right)$

$f_{3}=\frac{1}{2 L^{4}}\left(b^{2} \delta_{x}^{2}+a^{2} \delta_{y}^{2}\right) \varphi^{2}$
In that case $2 f_{3} \ll f_{1}^{2}$ and relation $\sqrt{1+\varepsilon} \approx 1+\frac{\varepsilon}{2}$ can be applied. By taking into account $\delta^{s}=L f_{1}$ stiffness (49) can be presented in the form

$\widetilde{K}_{R M S}=\widetilde{K}_{0}+\frac{C}{2 L^{4} \delta^{s}}\left(b^{2} \delta_{x}^{2}+a^{2} \delta_{y}^{2}\right) \varphi^{2}$.

The stiffness is the same in any direction, while that obtained by the energy approach is different, Eqs. (43)-(45). Actually, the constitutive quantities of (52) are spread into Eqs. (43)-(45) in a refined energetic way. Yaw influences translations and vice versa.

\section{Comparison analysis of different stiffness formulations}

\subsection{Uncoupled static analysis}

Influence of six restoring stiffness formulations presented in Sections $2-4$, on uncoupled static platform response, i.e. ignoring tendon influence, is analysed in the case of well known ISSC TLP, which is often used in the relevant literature as a benchmark [12]. The platform main particulars are listed in Table 1, where tension leg stiffness and mass parameters are related to the center of gravity.

Large surge and yaw displacements are given in order to magnify their coupling: $\delta_{x}=0.1 L=41.5 \mathrm{~m}$ and $\varphi=45^{\circ}=0.78537 \mathrm{rad}$. The surge force $F_{x}=\widetilde{K}_{0} \delta_{x}$ and yaw moment $M_{z}=r^{2} \widetilde{K}_{0} \varphi$, are calculated by employing formula (34) for $\widetilde{K}_{0}$. The partial setdown for surge and yaw are determined according to (25):

$\delta_{F}^{s}=\frac{1}{2 L} \delta_{x}^{2}=2.075 \mathrm{~m}$,

$\delta_{M}^{s}=\frac{r^{2}}{2 L} \varphi^{2}=2.748 \mathrm{~m}$

That leads to $F_{x}=1.563 \times 10^{4} \mathrm{kN}$ and $M_{z}=1.136 \times 10^{6} \mathrm{kN} \mathrm{m}$.

Furthermore, force $F_{x}$ and $M_{z}$ are imposed to the platform separately and than together in order to analyse interaction between surge and yaw. The obtained results for uncoupled and coupled case and six stiffness formulations, determined iteratively, are listed in Table 2. All formulations in case of $F_{x}$ give the same value for $\delta_{x}$ and $\delta^{s}$, respectively. Moment $M_{z}$ causes almost the same displacements $\varphi$ and $\delta^{s}$, for force equilibrium and energy balance. Differences of ca. $20 \%$ are caused by yaw treatment as a small or large quantity. Common action of $F_{x}$ and $M_{z}$ results with different values of all displacements, $\delta_{x}, \varphi, \delta^{s}$, for considered six cases, i.e. three different approaches and two yaw treatments.

In order to evaluate which of the above results are more realistic, the same nonlinear problem is solved by the finite element method by using program LS DYNA [13]. A quite simple FEM model is constructed as shown in Fig. 4. The platform is modeled as a thick plate of thickness $t=1 \mathrm{~m}$ and tendons by one finite element. Force $F_{x}$ is lumped in the plate corners, while moment $M_{z}$ is distrib-

Table 2

Platform displacements.

\begin{tabular}{|c|c|c|c|c|c|c|c|c|c|c|c|}
\hline \multirow[t]{2}{*}{ Case no. } & \multirow[t]{2}{*}{ Approach } & \multirow{2}{*}{$\begin{array}{l}\text { Averaging of tendon } \\
\text { forces }\end{array}$} & \multirow{2}{*}{$\begin{array}{l}\text { Yaw } \\
\text { treatment }\end{array}$} & \multirow{2}{*}{$\begin{array}{l}\text { Loading } \\
\text { condition } \\
\text { Ref. }\end{array}$} & \multicolumn{2}{|c|}{$F_{x}=1.563 \times 10^{4} \mathrm{kN}$} & \multicolumn{2}{|c|}{$M_{z}=1.136 \times 10^{6} \mathrm{kN} \mathrm{m}$} & \multicolumn{3}{|l|}{$F_{x} \& M_{z}$} \\
\hline & & & & & $\delta_{x}(\mathrm{~m})$ & $\delta^{s}(\mathrm{~m})$ & $\varphi\left(^{\circ}\right)$ & $\delta^{s}(\mathrm{~m})$ & $\delta_{x}(\mathrm{~m})$ & $\varphi\left(^{\circ}\right)$ & $\delta^{s}(\mathrm{~m})$ \\
\hline 1 & Force equilibrium & Arithmetic & Small & [9] Section 2.2 & 41.5 & 2.075 & 45 & 2.748 & 37.174 & 41.87 & 4.044 \\
\hline 2 & Force equilibrium & Arithmetic & Large & Section 2.1 & 41.5 & 2.075 & 50 & 3.392 & 36.604 & 46.01 & 4.336 \\
\hline 3 & Force equilibrium & RMS & Small & Section 4.2 & 41.5 & 2.075 & 45 & 2.748 & 36.110 & 40.67 & 3.815 \\
\hline 4 & Force equilibrium & RMS & Large & Section 4.1 & 41.5 & 2.075 & 50 & 3.392 & 35.596 & 44.40 & 4.071 \\
\hline 5 & Energy balance & Quadratic & Small & [10] Section 3.2 & 41.5 & 2.075 & 45 & 2.748 & 34.204 & 39.98 & 3.579 \\
\hline 6 & Energy balance & Quadratic & Large & Section 3.1 & 41.5 & 2.075 & 50 & 3.182 & 33.330 & 43.79 & 3.817 \\
\hline 7 & FEM & & & LS DYNA & 41.25 & 2.055 & 49.1 & 3.088 & 33.343 & 42.95 & 3.746 \\
\hline
\end{tabular}




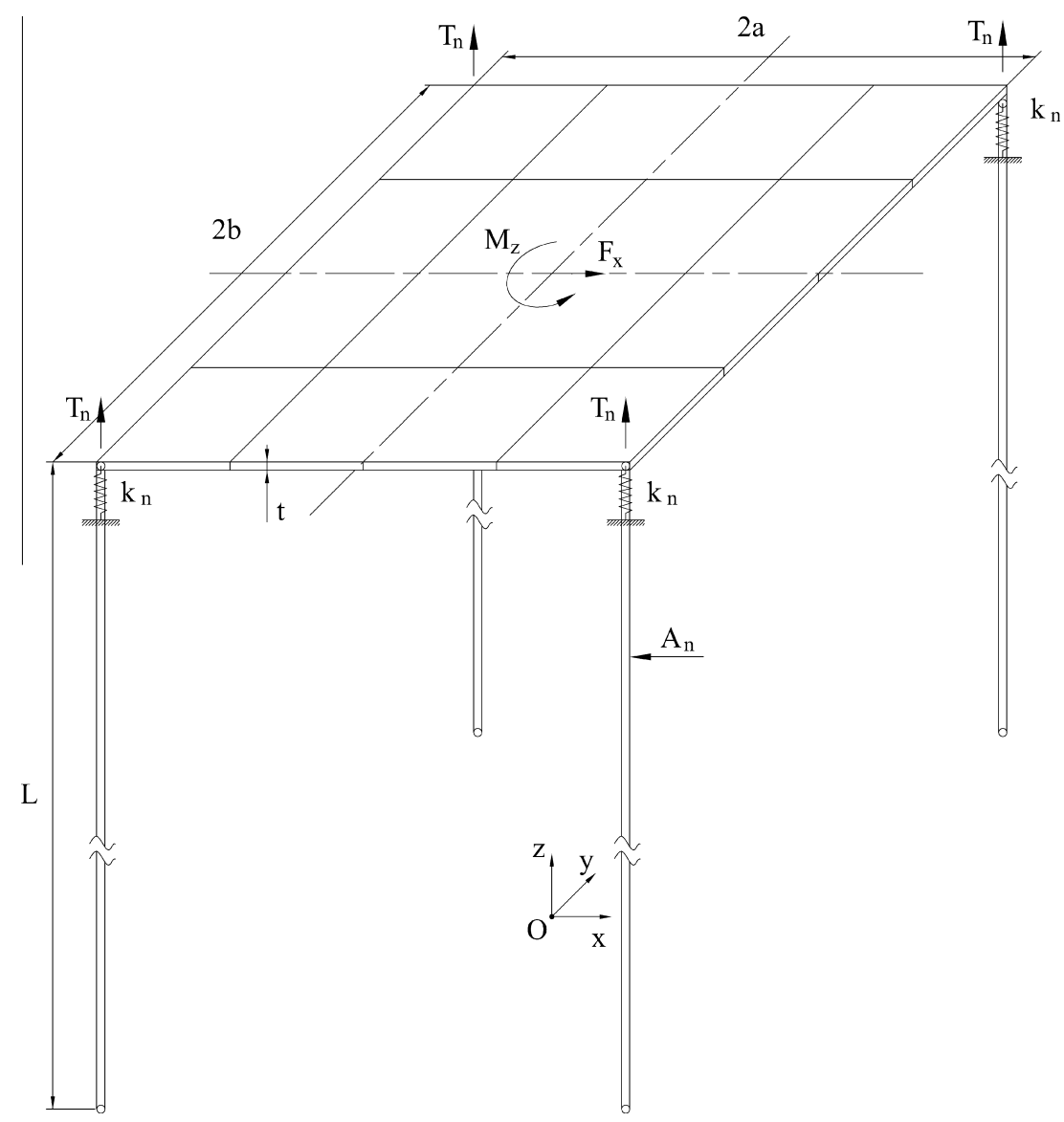

Fig. 4. TLP FEM model.

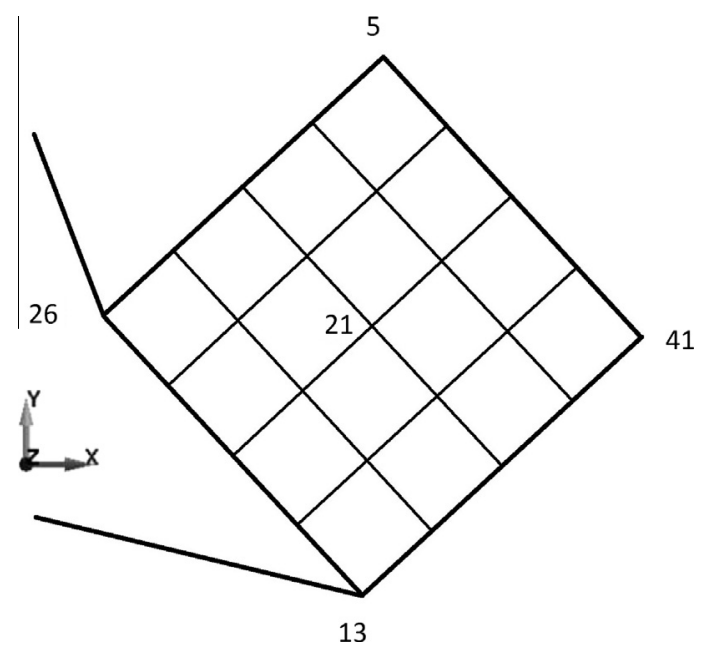

Fig. 5. Bird's view of platform in offset position.

uted in all 25 nodes. The hydrostatic springs in the plate corners take value $k_{n}=\frac{1}{4} \rho g A_{W L}=2192 \mathrm{kN} / \mathrm{m}$. Value of the Young's modulus is considerably increased $\left(E^{*}=10^{3} E\right)$ in order to constrain initial tendon strain due to imposed tendon pretension forces $T_{n}$, Fig. 4 .

Numerical calculation is performed separately for particular loads $F_{x}$ and $M_{z}$, and then for their common action. Due to geometric nonlinearity caused by the large displacements the static problem is solved as a dynamic one in the time domain by the step-bystep integration method and slowly increasing load values in order

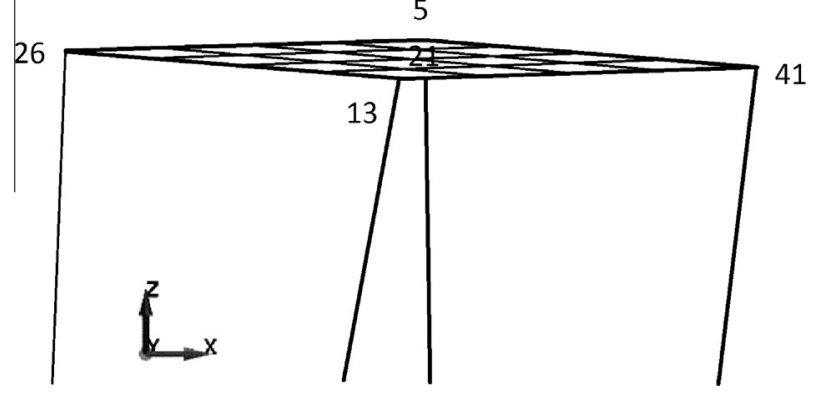

Fig. 6. Lateral view of platform in offset position.

to avoid inertia influence. First force $F_{x}$ is imposed and then moment $M_{z}$ so that their particular influence on response can be noticed. The bird's view of the platform in the equilibrated translated and rotated position is shown in Fig. 5. Figs. 6 and 7 show zoomed lateral and front view, where platform small pitch and roll can be noticed.

The time history of the platform longitudinal, transversal and vertical displacements is shown in Figs. 8-10, respectively. During $F_{x}$ action displacements of all platform corners are the same. By activating $M_{z}$ spreading of displacements due to rotation is noticeable. Displacement $\delta_{x}$ is slightly reduced, while setdown $\delta^{s}$ is considerably increased.

The FEM results for all three loading conditions are added in Table 2. Response due to $F_{x}$ is slightly smaller than the analytical values. In case of $M_{z}$ action numerical results are also somewhat lower than analytical ones for energy balance approach and yaw treat- 
26

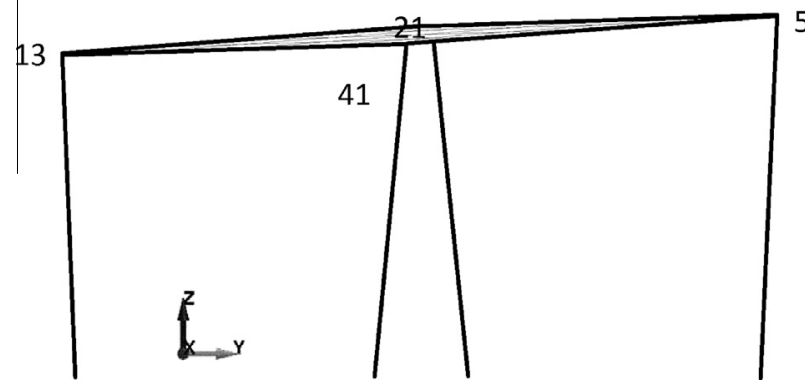

Fig. 7. Front view of platform in offset position.

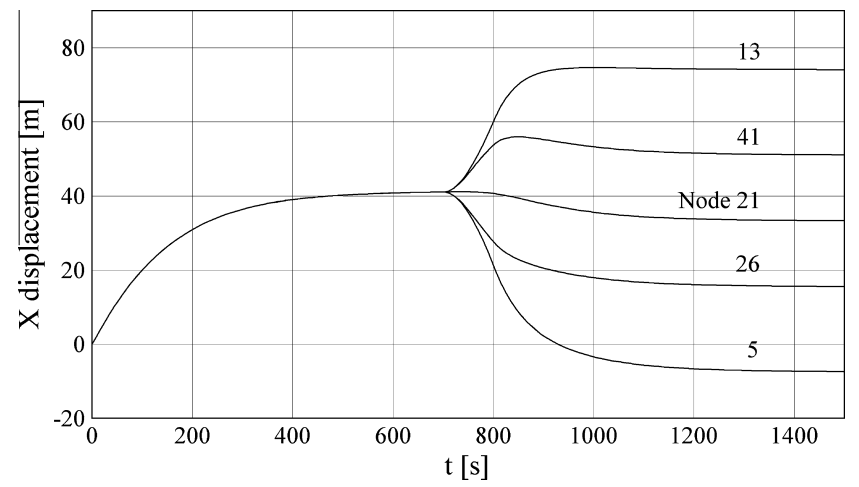

Fig. 8. Time history of platform longitudinal displacement.

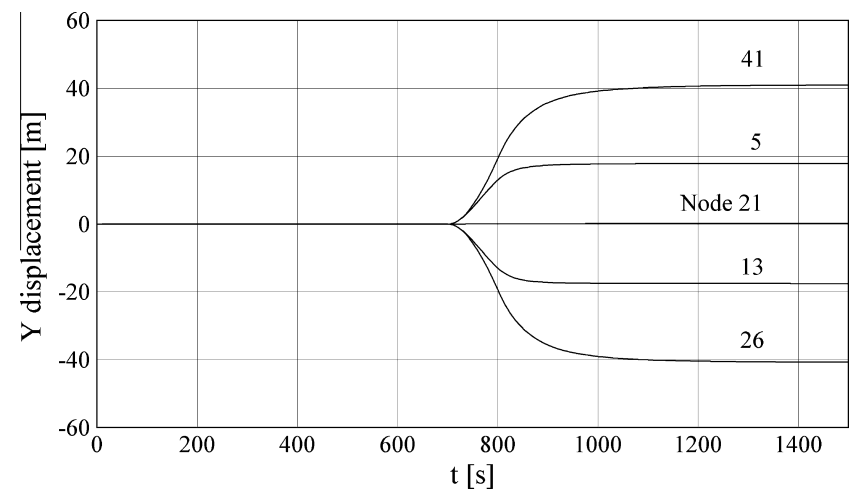

Fig. 9. Time history of platform transverse displacement.

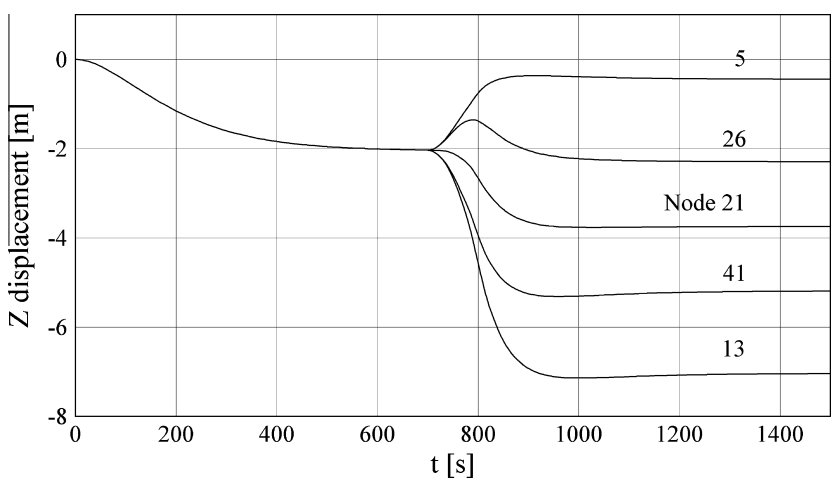

Fig. 10. Time history of platform setdown.

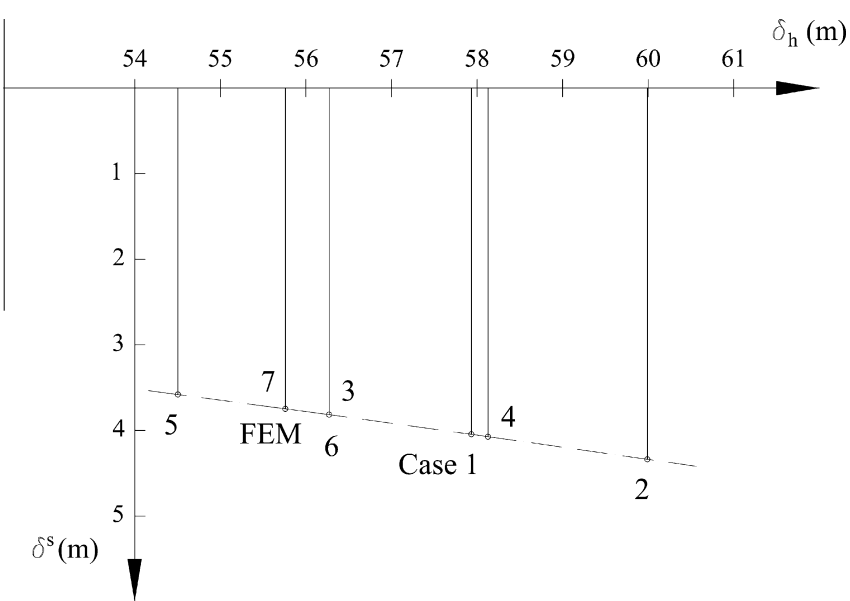

Fig. 11. Setdown versus virtual horizontal displacement.

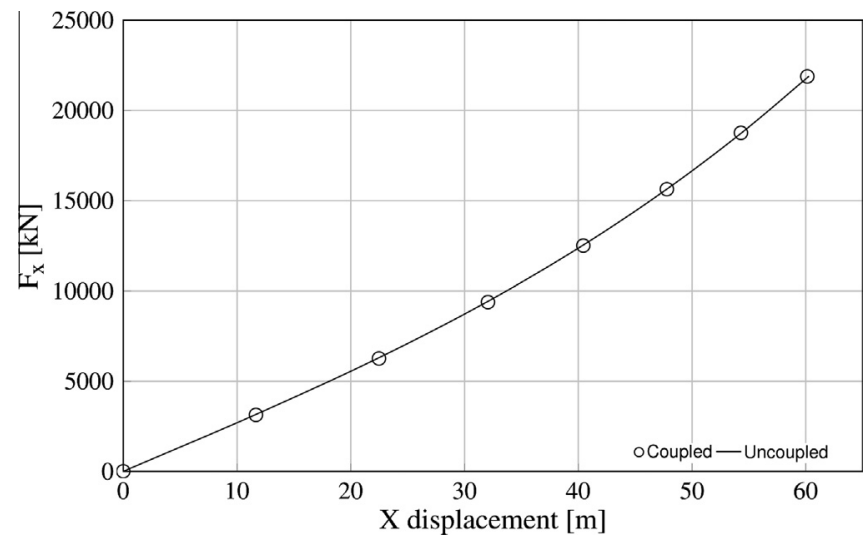

Fig. 12. Horizontal restoring force versus horizontal displacement.

ment as a large value. Since the analytical solutions for particular load are rigorous, they can be used for validation of the FEM results.

For simultaneous action of $F_{x}$ and $M_{z}$ the analytical results are nearly equal to the numerical ones. However, the latter are more accurate and therefore are used as referent values. Values of setdown in cases 3 and 6 are closer to the FEM ones, than those obtained for the other four cases. However, in case 6 better agreement for surge and yaw is obtained. Spreading of setdown $\delta^{s}$ as function of virtual horizontal displacement, $\delta_{h}=\sqrt{2 L \delta^{s}}$, which comprises both surge, $\delta_{x}$, and yaw, $\delta_{\varphi}$, Eq. (24), is shown in Fig. 11. The figure represents zoom of the complete diagram constructed in accordance with Fig. 3.

\subsection{Coupled static analysis}

The ISSC TLP is exposed to action of static surge force $F_{x}$, imposed at the pole level P, Fig. 1, by gradually increasing its magnitude. In analytical uncoupled analysis one half of the tendon weight is added to the platform bottom and another half to the sea bottom.

The same task is analysed as coupled platform and tendon problem by the finite element method, [14] and [15]. The platform is represented by one rigid plate element, while each tendon is modeled by four beam finite elements with realistic stiffness and distributed weight. 


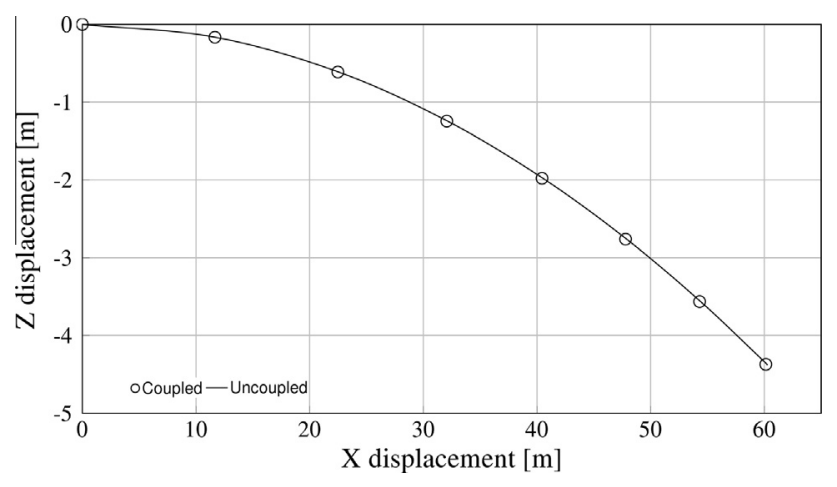

Fig. 13. Vertical displacement versus horizontal displacement.

Table 3

Comparison of coupled (FEM) and uncoupled (analytical) TLP static analysis.

\begin{tabular}{lcccrr}
\hline \multirow{2}{*}{$\mathrm{F}_{X}(\mathrm{kN})$} & Coupled & & \multicolumn{2}{c}{ Uncoupled } \\
\cline { 2 - 3 } \cline { 5 - 6 } & $X(\mathrm{~m})$ & $Z(\mathrm{~m})$ & & $X(\mathrm{~m})$ & \multicolumn{1}{c}{$Z(\mathrm{~m})$} \\
\hline 0 & 0.000 & 0.000 & 0.000 & 0.000 \\
3126 & 11.667 & -0.165 & 11.559 & -0.161 \\
6252 & 22.482 & -0.613 & 22.327 & -0.601 \\
9378 & 32.066 & -1.245 & 31.926 & -1.228 \\
12,504 & 40.444 & -1.979 & 40.355 & -1.962 \\
15,630 & 47.793 & -2.763 & 47.770 & -2.749 \\
18,756 & 54.304 & -3.565 & 54.350 & -3.559 \\
21,882 & 60.135 & -4.372 & 60.251 & -4.374 \\
\hline
\end{tabular}

The obtained results are shown in Figs. 12 and 13. The former represents surge as function of imposed force $F_{x}$, and latter shows vertical displacement as function of surge. Difference between analytical uncoupled and FEM coupled analysis is very small as can be noticed in Table 3. In uncoupled analysis vertical displacement is pure setdown, while in coupled analysis there is small release due to tendon elasticity. Since results of uncoupled and coupled analysis are very close, one can conclude that the former is reliable enough for the static analysis.

\section{Conclusion}

Nonlinear restoring stiffness plays very important role in TLPS dynamic behavior. The traditionally used stiffness matrix manifests shortcomings $[7,8]$, while there is doubt concerning consistency of the recently derived matrix [10]. Therefore a detailed analysis of the problem is undertaken. Both the force equilibrium approach, with algebraic mean and RMS of tendon forces, and potential energy approach with quadratic mean are used and new stiffness matrices, treating yaw as a small and large displacement, are derived. Comparison of numerical results determined for ISSC TLP with those of FEM analysis shows that the stiffness matrix formulation based on energy approach for large yaw is the most reliable.

The derived secant stiffness matrix for horizontal motions has to be completed with terms for vertical motions, i.e. heave, pitch and roll. Such matrix is diagonal since it is established with respect to the pole P, Fig. 1, while the accompanying mass matrix is not diagonal. Setdown, as a slave d.o.f. of the master horizontal displacements, induces vertical inertia force, which has to be incorporated in the mass matrix. However, it is easier to add setdown to heave forming in such a way complete vertical motion. The stiffness matrix is accordingly transformed; it becomes asymmetric [9]. In order to perform dynamic analysis in ordinary way with respect to the center of gravity, both matrices have to be transformed from the tendon natural coordinate system to the global system with origin in the center of gravity.

Some software operate with nonlinear restoring forces and some with their increments, i.e. secant and tangent stiffness matrix, respectively. Both matrices can be easily incorporated in the existing computer codes. In that way applicability and accuracy of uncoupled model is extended and increased, respectively. That might be interesting for improvement of the required uncoupled time domain model in the Classification Rules for design and construction of TLPs, [16].

\section{Acknowledgement}

The authors express their gratitude to Dr. Ivan Ćatipović from the University of Zagreb for the coupled static analysis of TLP by the finite element method.

\section{Appendix A. Potential energy based on equilibrated forces}

After the nonlinear stiffness for large translation and small rotation and algebraic mean of tendon forces based on equilibrium of internal and external forces is established, it is interesting to formulate potential energy of the considered elastic system. The external forces are specified in simple form

$F_{x}=\widetilde{K}_{0} \delta_{x}, \quad F_{y}=\widetilde{K}_{0} \delta_{y}, \quad M_{z}=r^{2} \widetilde{K}_{0} \varphi$,

where $\widetilde{K}_{0}$ is common stiffness, Eq. (18). Since $L_{z}=L-\delta^{s}$, stiffness, Eq. (18) can be expressed as function of setdown

$\widetilde{K}_{0}=\frac{C}{L-\delta^{s}}-\rho g A_{W L}$.

Work of the horizontal forces is

$V=\int F_{x} \mathrm{~d} \delta_{x}+\int F_{y} \mathrm{~d} \delta_{y}+\int M_{z} \mathrm{~d} \varphi$

and by substituting (A1) into (A3) yields

$V=\int \widetilde{K}_{0}\left(\delta_{x} \mathrm{~d} \delta_{x}+\delta_{y} \mathrm{~d} \delta_{y}+r^{2} \varphi \mathrm{d} \varphi\right)$.

Expression in the brackets, according to (25), represents the total differential of setdown, i.e. (..) $=L \mathrm{~d} \delta^{s}$. Hence, by substituting (18) into (A4) one finds

$$
\begin{aligned}
V & =L \int\left(\frac{C}{L-\delta^{s}}-\rho g A_{W L}\right) \mathrm{d}\left(\delta^{s}\right) \\
& =-L C \ln \left(1-\frac{\delta^{s}}{L}\right)-\rho g A_{W L} L \delta^{s} .
\end{aligned}
$$

The logarithmic function can be expanded into the power series

$\ln \left(1-\frac{\delta^{s}}{L}\right)=-\sum_{k=1}^{\infty} \frac{1}{k}\left(\frac{\delta^{s}}{L}\right)^{k}$,

that leads to

$V=T \delta^{s}+\frac{C}{2 L}\left(\delta^{s}\right)^{2}+L C \sum_{k=3}^{\infty} \frac{1}{k}\left(\frac{\delta^{s}}{L}\right)^{k}$,

where the first two term of the series are expressed explicitly. Quantities $C$ and $\delta^{s}$ are specified by Eqs. (19) and (25), respectively. According to (12) $\delta^{s}=L f_{1}$ and the potential energy can be presented in the following form

$V=T L f_{1}+\frac{1}{2} C L f_{1}^{2}+L C \sum_{k=3}^{\infty} \frac{1}{k} f_{1}^{k}$.

By comparing (A8) with (33) it is obvious that the first two terms are identical. The third term in (33) is of the same order of magni- 
tude as the second one, and cannot be compensated with the third term in (A8), since it is a small quantity of higher order.

Formulation of potential energy (A8) leads to the stiffness

$\widetilde{K}_{0} \approx \frac{T}{L}+\frac{C}{L^{2}} \delta^{s}$,

which is common for any direction. The first and the second term in (A9) represent linear and nonlinear stiffness, respectively, [9].

\section{References}

[1] Chakrabarti S. Handbook of offshore engineering. Elsevier Science; 2005.

[2] Planning, designing and constructing tension leg platforms. API recommended practice 2T, 3rd ed. American Petroleum Institute; 2010.

[3] Adrezin R, Bar-Avi P, Benaroya H. Dynamic response of compliant offshore structures - review. J Aerosp Eng 1996;9(4):114-31.

[4] Faltinsen OM, Demirbilek Z. Hydrodynamic analysis of TLPs. In: Demirbilek Z editor. Tension leg platform: a state-of-the-art review. ASCE; 1989. p. 36-63.

[5] DNV - OS - C105 Structural design of TLP (LRFD method). Det Norske Veritas; 2008.

[6] Morgan J, Malaeb D. Dynamic analysis of tension leg platforms. In: Proceedings of the second international offshore mechanics and arctic engineering symposium; 1983. p. 31-7.
[7] Jain AK, Nonlinear coupled response of offshore tension leg platform to regular wave forces. Ocean Eng 1997;24(7):577-93.

[8] Chandrasekaran S, Jain AK. Dynamic behavior of square and triangular offshore tension leg platforms under regular wave load. Ocean Eng 2002;29(3):279-313.

[9] Senjanović I, Tomić M, Hadžić N. Formulation of consistent nonlinear restoring stiffness for dynamic analysis of tension leg platform and its influence on response. Marine Struct 2013;30(1):1-32.

[10] Low YM. Frequency domain analysis of a tension leg platform with statistical linearization of the tendon restoring forces. Marine Struct 2009;22(3):480-503.

[11] Murray J, Yang CK, Yang W, Krishnaswamy P, Zou J. An extended tension leg platform design for Post-Katrina Gulf of Mexico. In: The proceedings of the nineteenth international offshore and polar engineering conference (ISOPE), vol. 1 ; 2009. p. 120-7.

[12] Taylor RE, Jefferys ER. Variability of hydrodynamic load predictions for a tension leg platform. Ocean Eng 1986;13(5):449-90.

[13] LS DYNA theory manual. Livermore Software Technology Corporation; 2006.

[14] Garrett DL. Dynamic analysis of slender rods. J Energy Resour Technol 1982;104(4):302-6.

[15] Ćatipović I, Čorić V, Vukčević V. Dynamics of FPSO with polyester mooring lines. In; Proceedings of the twenty-second international offshore and polar engineering conference; 2012. p. 996-1003.

[16] Rules for the classification of tension leg platforms (TLP). Bureau Veritas; 2012. 